\title{
Procrastinación general y académica en una muestra de estudiantes de secundaria de Lima metropolitana
}

\author{
Óscar Ricardo Álvarez Blas \\ Universidad de Lima
}

Recibido: 1 de abril del 2010 / Aprobado: 26 de mayo del 2010

Se estudió la presencia de la procrastinación general y académica en un grupo de estudiantes de secundaria $(N=235)$ de ambos géneros pertenecientes a colegios no estatales de Lima metropolitana, para lo cual se utilizó la Escala de Procrastinación General y Académica (Busko, 1998). Sobre la base del análisis de los resultados se encontró que la procrastinación académica era mayor que la general, y no existian diferencias significativas según rol genérico y el grado de estudios. Se adjunta el instrumento utilizado, el cual fue adaptado del inglés al español por el autor del presente trabajo, con la intención de que sirva para futuras investigaciones.

procrastinación / procrastinación general / procrastinación académica

\section{General and academic procrastination in a sample of secondary students in metropolitan Lima}

General and academic procrastination was studied in a group of secondary students $(N=235)$ of both genders in several private high schools in metropolitan Lima. The sample was assessed using the General and Academic Procrastination Scale (Busko, 1998). Results show that academic procrastination is greater than general procrastination, and there are no significant differences depending on gender role or level of study. For further research, it is recommended not to take into the sample students from consecutive grades of study (in the present study, students were juniors and seniors) to better understand the effects of socialization in a longer period of time.

procrastination / general procrastination / academic procrastination 


\section{INTRODUCCIÓN}

La procrastinación es la tendencia a posponer o retrasar la finalización de una labor evitando las responsabilidades, decisiones y tareas que requieren ser desarrolladas (Haycock, McCarthy \& Skay, 1998; citado por Steel, 2007).

A esta tendencia de conducta no se le debe percibir como un simple error o un mal cualquiera, sino como uno de los múltiples casos en que las personas no se enfocan ni se orientan a alcanzar sus intereses en forma eficiente y productiva.

En este sentido, no solo se toma en cuenta el hecho de postergar o dejar para después las tareas que pueden ser desarrolladas al momento, sino que se pierde la esencia de la importancia que tiene realizar tareas o actividades en forma planificada y ordenada, obteniendo así mayores beneficios y satisfacciones para la consecución de objetivos tanto a corto como a mediano plazo. Ello finalmente involucra logros que pudiesen ser importantes para las personas, en este caso estudiantes de cuarto y quinto de secundaria de colegios no estatales de Lima.

La importancia de estudiar la procrastinación en estudiantes de cuarto y quinto de secundaria, radica en que este fenómeno es uno de los problemas que actúan en contra del buen aprendizaje y desempeño académico de los estudiantes de colegios, pues al estar estos alumnos en un periodo crítico y difícil de sus vidas, tienden a postergar sus responsabilidades académicas. Así, de acuerdo con Ferrari (1995) las diversas formas mediante las cuales los alumnos procrastinan incluyen la dificultad en el cumplimiento de horarios, entrega de asignaciones fuera de fecha, retraso para desarrollar tareas y esperar hasta el último minuto para finalizar sus labores, entre otras.

\section{Procrastinación}

Las sociedades avanzadas, la tecnología, y por lo tanto la modernidad, obligan a las personas a ocuparse de trivialidades, a vivir de prisa y a enfocarse en aspectos que impiden su completo desarrollo, lo que lleva a una falta y mala distribución del tiempo, que finalmente interfieren con el logro de objetivos que pudiesen trazarse las personas.

El término 'procastinación' proviene del latín procrastinare, que literalmente significa dejar las cosas o posponerlas para otro día (DeSimone, 1993; citado por Ferrari et al., 1995).

Para Ferrari, Johnson y McCown (1995) la procrastinación es un constructo que ha existido a lo largo de la vida, sin embargo, la connotación negativa surge a partir de la revolución industrial.

DeSimone (1993; como cita Ferrari et al., 1995) notó que muchas de las sociedades preindustrializadas no tenían términos comparables con la noción de procrastinación. 
Así, los antiguos egipcios, por ejemplo, poseían dos verbos que fueron traducidos como significante de procrastinación. Uno denotaba la útil costumbre de evitar el trabajo y esfuerzo impulsivo, mientras que la otra, el pernicioso hábito de la pereza en la realización de una tarea necesaria para la subsistencia, tales como el cultivar el campo en el tiempo apropiado del año. Por otro lado, para los romanos la procrastinación reflejaba la noción juiciosa y sabia de espera, de forma que se reconocía el momento idóneo para contemplar la presencia de los enemigos en los conflictos militares.

Desde una perspectiva psicodinámica, la procrastinación estaría vinculada al concepto de evitación de tareas específicas, el cual fue discutido por Freud en el año 1926. De esta manera, se hace notar el rol de la angustia al evitar una tarea, explicado por Freud en Inhibiciones, sintoma y angustia (Freud, 1926; citado por Ferrari et al., 1995), en donde menciona que esta es tomada como una alerta para el yo, al señalarle el material inconscientemente reprimido que trata de emerger de modo amenazante; por lo tanto, una vez que esta angustia es detectada, el yo emplea una variedad de mecanismos de defensa que le permitan, en algunos casos, evitar la tarea.

Burka \& Yuen (1983; citados por Steel, 2007) señalan que la procrastinación tiene sus principios en el miedo que siente el niño de que sus actos lleven a consecuencias no positivas, sea estable- ciendo un vínculo que no tiene la capacidad de retener o alejándolo del objeto. De adulto, el riesgo de pérdida o incapacidad lo lleva a protegerse del temor a fallar, temor a tener éxito, temor a la separación o al compromiso, postergando sus acciones continuamente.

La visión conductual refiere que una conducta se mantiene cuando es reforzada, es decir, las conductas persisten por sus consecuencias (Skinner, 1977). Por tal razón, las personas procrastinan porque sus conductas de postergación propiamente han sido reforzadas y además han tenido éxito debido a diversos factores propios del ambiente, que han permitido continuar con este tipo de acciones.

Mientras la visión cognitiva-conductual, Ellis y Knaus (1977) refieren que la procrastinación se debe a que la persona posee creencias irracionales sobre lo que implica terminar una tarea adecuadamente, en ese sentido, tiende a plantearse metas altas y poco reales que por lo general lo llevan al fracaso. Es entonces que al intentar calmar esas consecuencias emocionales, demoran el inicio de las tareas hasta que no sea factible completarlas de manera apropiada, evitando así cuestionar sus habilidades y capacidades.

\section{Procrastinación general}

La procrastinación es un constructo polisémico, estudiado y desarrollado por muchos, los que tienden a presentar 
múltiples definiciones agrupando un elemento común, el aplazamiento o la demora.

Ferrari et al. (1995) la definen como el hecho de posponer la culminación de una tarea que usualmente resulta en un estado de insatisfacción o malestar subjetivo (Burka \& Yuen, 1983; citado por Ferrari et al., 1995). Así, la procrastinación debe ser considerada como el obstáculo que imposibilita a las personas conseguir sus intereses de forma eficiente y productiva, representando una disociación entre habilidades y capacidades para afrontar apropiadamente sus tareas y deberes.

Popoola (2005; citado por Akinsola, 2007) considera la procrastinación como un rasgo que tiene componentes cognitivos, conductuales y emocionales. Noran (2000; citado por Akinsola, 2007) la define como el evitar la realización de una tarea que requiere ser finalizada. Menciona que algunos se inclinan por pasar el tiempo socializando, en lugar de desarrollar una asignación importante que debe presentarse, $\mathrm{y}$ otros prefieren ir al cine o ver una película antes que estudiar para un examen. De esta manera, refiere que el procrastinador es aquel que sabe lo que quiere hacer, en cierta manera, que sabe que puede y que trata de hacerlo, pero que en realidad no lo hace.

Burka y Yuen (1982; citado por Solomon \& Rothblum, 1984) manifiesta que quienes tienen problemas con la procrastinación, por lo general lo atri- buyen a dificultades de personalidad, tales como flojera, indisciplina o no saber cómo organizar el tiempo.

Finalmente, personas que reportan frecuente procrastinación están comprometidas en conductas de autosabotaje (Ferrari \& Tice, 2000; citado por Ferrari \& Díaz-Morales, 2007); excusas falsas (Ferrari, 1993; citado por Steel, 2007); pobre autorregulación de su desempeño dentro de un periodo de tiempo (Ferrari, 2001; citado por Steel, 2007), y atribuyen la demora de tareas a factores distintos de su propio desempeño (Ferrari et al., 1995).

\section{Procrastinación ACADÉMICA}

Sabiendo que la procrastinación es definida como la tendencia a dejar de lado actividades hasta una futura fecha, las investigaciones señalan que al menos un $25 \%$ de estudiantes presentan experiencias de problemas de este tipo (Ellis \& Knaus, 1977; Solomon \& Rothblum, 1984), y esa procrastinación es frecuentemente asociada con negativas consecuencias académicas (Rothblum, Solomon \& Murakami, 1986). Por ello, también está relacionada con bajas notas y abandono de cursos (Semb, Glick \& Spencer, 1979; citado por LaForge, 2005).

La mayoría de estudios sobre procrastinación se han realizado en universitarios, enfocándose en la finalización de tareas de cursos y evaluaciones. De acuerdo con Solomon y Rothblum 
(1984) la procrastinación académica implica la experiencia de ansiedad debido a la persistente demora en las tareas académicas.

Otros autores, como Lay y Silverman (1996; citado por Wolters, 2003), destacan ese abatimiento en lugar de la ansiedad como motivador de la procrastinación. Johnson y Bloom (1995; citado por Balkis, 2009) señalan que la procrastinación ha sido estudiada a través de dos diferentes líneas de investigación: la primera, al indagar su relación con resultados y cumplimiento de metas, y la segunda, con respecto a los factores de personalidad.

Lo que más se observa en estudiantes procrastinadores es que empiezan a estudiar mucho más tarde de lo óptimo. Es probable que este retraso se presente por la discordancia de su conducta o hábito de estudio y sus intenciones (Milgram, Sroloff \& Rosenbaum, 1988; citados por Blunt, 1998), pero también porque su intención de estudiar está demorada. En consecuencia, estos se distraen fácilmente con otras actividades que no son las escolares, como por ejemplo las actividades sociales.

Rothblum, Solomon y Murakami (1986) definen la procrastinación académica como a) la tendencia a aplazar siempre o casi siempre una actividad académica, y b) siempre o casi siempre experimentar ansiedad asociada a la procrastinación. Asimismo, los procrastinadores muestran una alta posibi- lidad de estar fuera de fecha en sus tareas (Lay, 1990; citado por Blunt, 1998). Migram, Batin y Mower (1993; citado por Akinsola, 2007) señalan que una forma común de procrastinación académica en estudiantes, es esperar hasta el último minuto para empezar a desarrollar sus actividades o estudiar para un examen.

\section{HIPÓTESIS}

Para el desarrollo del estudio se plantearon las siguientes hipótesis:

\section{Hipótesis general}

Existen diferencias significativas en la procrastinación general y académica según rol genérico y grado de estudio.

\section{Hipótesis específicas}

- $\mathrm{H}_{1}$ : Existen diferencias entre hombres y mujeres en la procrastinación general.

- $\mathrm{H}_{2}$ : Existen diferencias entre hombres y mujeres en la procrastinación académica.

- $\mathrm{H}_{3}$ : Existen diferencias entre los alumnos de cuarto año y quinto año de secundaria en la procrastinación general.

- $\mathrm{H}_{4}$ : Existen diferencias entre alumnos de cuarto año y quinto año de secundaria en la procrastinación académica. 


\section{MÉTOdo}

El presente estudio fue de tipo descriptivo, pues para Dahnke (1989; citado en Hernández, Fernández \& Baptista, 2006), el propósito de estos estudios es especificar las propiedades, las características y los perfiles importantes de personas, grupos, comunidades o cualquier otro fenómeno que se someta al análisis. Es decir, miden, evalúan o recolectan datos sobre diversos conceptos (rol genérico y grado de estudio), aspectos, dimensiones o componentes del fenómeno por investigar, teniendo como objetivo la medición o recolección de información de manera independiente o conjunta de los conceptos o variables a los que se refieren, esto es, no indicando cómo se relacionan las variables medidas. El diseño fue de tipo descriptivo comparativo, pues este diseño tiene como objetivo indagar la incidencia de las modalidades o niveles de una o más variables en una población (Hernández, Fernández \& Baptista, 2006).

\section{Participantes}

Se consideraron estudiantes de ambos roles genéricos, que se encontraban cursando estudios de cuarto año y quinto año de secundaria en un grupo de colegios no estatales de Lima metropolitana. La muestra del estudio se obtuvo por un procedimiento no probabilístico de tipo intencional. La muestra total estuvo conformada por 235 estudiantes, de los cuales $80(34 \%)$ cursaban el cuarto de secundaria, y 155 (66\%) el quinto de secundaria. Según el género, 132 $(56,2 \%)$ eran hombres y $103(43,8 \%)$ mujeres (véase la tabla 1).

\section{Tabla 1}

Composición de la muestra por colegio, rol genérico y año de estudio

\begin{tabular}{lcc}
\hline Variables & Frecuencia & Porcentaje \\
\hline $\begin{array}{l}\text { Colegio } \\
\text { Uno }\end{array}$ & 26 & 11,1 \\
Dos & 119 & 50,6 \\
Tres & 90 & 38,3 \\
Rol genérico & & \\
$\quad$ Hombre & 132 & 56,2 \\
Mujer & 103 & 43,8 \\
Año de estudio & & \\
Cuarto & 80 & 34,0 \\
Quinto & 155 & 66,0 \\
\hline
\end{tabular}

$\mathrm{n}=235$

VARIABLES DE ESTUDIO

\section{Variables dependientes}

a) Procrastinación general: Tendencia a demorar o evitar tareas en general que deben ser completadas en cierto periodo de tiempo.

b) Procrastinación académica: Tendencia de siempre o casi siempre posponer tareas académicas que se le asignan a los alumnos. 
Variables independientes asignadas

a) Rol genérico: Hombres y mujeres.

b) Grado de estudio: Alumnos de cuarto y quinto de secundaria

\section{INSTRUMENTOS}

Escala de Procrastinación General y Académica de Busko (1998)

a) Escala de Procrastinación General (EPG).- Fue desarrollada por Busko (1998) para estudiar las causas y consecuencias del perfeccionismo y la procrastinación.

El instrumento original se aplicó a 112 estudiantes de pregrado de la Universidad de Guelph (33 hombres y 79 mujeres). La edad promedio fue de 22,36 años y los alumnos pertenecían al primero y al tercer ciclos de Ciencias Sociales, con una cantidad de 59 y 53 estudiantes, respectivamente. Así, por su construcción y redacción, el mismo puede ser aplicado a adolescentes.

Busko (1998) reportó que para evaluar la confiabilidad se utilizó la consistencia interna a través del coeficiente Alfa de Cronbach. Los resultados indican que se obtuvo un coeficiente alfa para la procrastinación general de 0.82 y de 0.86 para la académica. Los errores de medición obtenidos alcanzaron valores de 0.191 y 0.123 , respectivamente. La escala se compone de dos subescalas: Escala de Procrastinación
General y Escala de Procrastinación Académica, las que fueron traducidas y adaptadas al español para la presente investigación.

Esta Escala puede ser administrada en forma individual o colectiva. Normalmente, contestar el cuestionario toma entre 7 y 10 minutos y el tiempo total de aplicación del objetivo de la prueba no suele superar los 15 minutos. Está conformada por 13 reactivos, todos cerrados, permitiendo así evaluar con fiabilidad la tendencia hacia la procrastinación general. Todos los reactivos se puntúan mediante una escala de tipo Likert de cinco puntos.

1. SIEMPRE (Me ocurre siempre).

2. CASI SIEMPRE (Me ocurre mucho).

3. A VECES (Me ocurre alguna vez).

4. POCAS VECES (Me ocurre pocas veces o casi nunca).

5. NUNCA (No me ocurre nunca)

b) Escala de Procrastinación Académica (EPA).- Puede ser aplicada en forma individual o colectiva. Normalmente, contestar el cuestionario toma entre 8 y 12 minutos y el tiempo total de aplicación del objetivo de la prueba no suele superar los 17 minutos. Está compuesta por 16 reactivos, todos cerrados, permitiendo así evaluar con fiabilidad la tendencia hacia la procrastinación académica. Todos los reactivos se 
puntúan mediante una escala de tipo Likert de cinco puntos.

1. SIEMPRE (Me ocurre siempre).

2. CASI SIEMPRE (Me ocurre mucho).

3. A VECES (Me ocurre alguna vez).

4. POCAS VECES (Me ocurre pocas veces o casi nunca).

5. NUNCA (No me ocurre nunca).

\section{Desarrollo de la adaptación de las escalas}

Los pasos que se siguieron para la adaptación de los instrumentos desarrollados por Busko (1998) fueron los siguientes:

a) Traducción de ambas escalas al castellano; para ello, se solicitó la colaboración de un traductor y dos estudiantes de los últimos ciclos de traducción. Asimismo, se recibió apoyo de dos psicólogos para que revisaran la redacción de cada ítem para eliminar ambigüedades o confusiones.

b) Una vez hechas las correcciones necesarias, se procedió a contactar con los colegios seleccionados, y en coordinación con las autoridades correspondientes se solicitaron las primeras horas de la mañana para realizar las evaluaciones.

c) La aplicación del instrumento fue grupal bajo la dirección del inves- tigador, en el transcurso de un mes; el tiempo aproximado para responder las dos escalas fue de 20 a 22 minutos.

d) Se aplicó un registro anecdótico para anotar aquellos inconvenientes que pudiesen suscitarse, sin embargo, no hubo tales dificultades, al no surgir preguntas ni dudas durante la aplicación de las escalas.

e) Finalmente, se realizó el análisis estadístico para indagar la confiabilidad y validez de las escalas. Los detalles de dicho análisis se presen$\tan$ en el siguiente rubro.

\section{Resultados}

\section{Análisis psicométrico de las escalas}

Escala de Procrastinación General

Los resultados del análisis psicométrico de la Escala de Procrastinación General (véase tabla 2), permiten apreciar que los ítems fluctúan entre 0.26 (ítem 4) y 0.65 (ítem 8 ), los cuales son estadísticamente significativos, y superan el criterio de ser mayores de 0.20 (Kline, 1995), con lo cual se concluye que todos los ítems son aceptados.

En el caso de la confiabilidad se observa que el coeficiente de consistencia interna Alfa de Cronbach asciende a 0.87 , lo que permite concluir que la Escala de Procrastinación General presenta confiabilidad. 
Tabla 2

Análisis de la confiabilidad de la EPG

\begin{tabular}{cccc}
\hline Ítem & M & D. E. & .ritc \\
\hline Ítem 1 & 3.17 & 0.78 & $0.45^{*}$ \\
Ítem 2 & 2.67 & 0.95 & $0.50^{*}$ \\
Ítem 3 & 3.34 & 0.94 & $0.42^{*}$ \\
Ítem 4 & 2.65 & 0.99 & $0.26^{*}$ \\
Ítem 5 & 3.74 & 0.99 & $0.50^{*}$ \\
Ítem 6 & 3.10 & 0.88 & $0.50^{*}$ \\
Ítem 7 & 3.12 & 0.94 & $0.57^{*}$ \\
Ítem 8 & 3.17 & 1.01 & $0.65^{*}$ \\
Ítem 9 & 2.74 & 0.91 & $0.60^{*}$ \\
Ítem 10 & 2.83 & 0.92 & $0.51^{*}$ \\
Ítem 11 & 2.82 & 1.07 & $0.55^{*}$ \\
Ítem 12 & 2.98 & 1.06 & $0.51^{*}$ \\
Ítem 13 & 1.92 & 0.86 & $0.52^{*}$ \\
\hline
\end{tabular}

Alfa de Cronbach $\mathbf{= 0 . 8 7}$

${ }^{*} \mathrm{p}<.05$

$\mathrm{n}=235$

El análisis de la validez de constructo de la EPG, realizado a través del análisis factorial exploratorio (véase la tabla 3). En primer lugar, se analizó si los datos presentaban las condiciones necesarias para proceder con el análisis factorial, a través de los siguientes estadísticos:

a) La medida de adecuación del muestreo de Kaiser-Meyer-Olkin alcanza un valor de 0.82 , lo cual indica que las variables tienen un adecuado potencial explicativo. b) El test de esfericidad de Bartlett presenta un valor que es significativo $($ Chi-cuadrado $=716.83 \mathrm{p}<.05)$, lo cual corrobora que los coeficientes de correlación entre los ítems son lo suficientemente elevados como para continuar con el análisis factorial.

El análisis factorial efectuado a través del método de los componentes principales indica que existe un solo factor que permite explicar el $30,99 \%$ de la varianza total. Los resultados alcanzados permiten concluir que la escala presenta validez de constructo. 
Tabla 3

Análisis de la validez de constructo de la EPG a través del análisis factorial exploratorio

\begin{tabular}{lccc}
\hline Ítem & M & D. E. & Factor 1 \\
\hline Ítem 1 & 3.17 & 0.78 & 0.59 \\
Ítem 2 & 2.67 & 0.95 & 0.63 \\
Ítem 3 & 3.34 & 0.94 & 0.56 \\
Ítem 4 & 2.65 & 0.99 & 0.32 \\
Ítem 5 & 3.74 & 0.99 & 0.30 \\
Ítem 6 & 3.10 & 0.88 & 0.63 \\
Ítem 7 & 3.12 & 0.94 & 0.70 \\
Ítem 8 & 3.17 & 1.01 & 0.35 \\
Ítem 9 & 2.74 & 0.91 & 0.71 \\
Ítem 10 & 2.83 & 0.92 & 0.62 \\
Ítem 11 & 2.82 & 1.07 & 0.66 \\
Ítem 12 & 2.98 & 1.06 & 0.63 \\
Ítem 13 & 1.92 & 0.87 & 0.38 \\
\hline
\end{tabular}

Determinante $=0.044$

Medida de adecuación muestral de Kaiser-Meyer-Olkin. $=0.823$

Prueba de esfericidad de Bartlett Chi-cuadrado $=716.83$ G. L. $=78 p=.0001$

$\mathrm{N}=235$

Tabla 4

Análisis de la confiabilidad de la EPA

\begin{tabular}{lccc}
\hline Ítem & $\mathbf{M}$ & $\mathbf{D . ~ E . ~}$ & ritc \\
\hline Ítem 1 & 3.05 & 0.88 & $0.43^{*}$ \\
Ítem 2 & 2.74 & 0.88 & $0.51^{*}$ \\
Ítem 3 & 2.95 & 1.04 & $0.47^{*}$ \\
Ítem 4 & 2.83 & 1.09 & $0.47^{*}$ \\
Ítem 5 & 3.43 & 0.96 & $0.47^{*}$ \\
Ítem 6 & 4.40 & 0.92 & $0.41^{*}$ \\
Ítem 7 & 3.58 & 0.85 & $0.31^{*}$ \\
Ítem 8 & 2.95 & 0.99 & $0.47^{*}$ \\
Ítem 9 & 2.92 & 1.05 & $0.54^{*}$ \\
Ítem 10 & 3.44 & 0.95 & $0.34^{*}$ \\
Ítem 11 & 3.03 & 0.94 & $0.45^{*}$ \\
Ítem 12 & 3.38 & 0.91 & $0.40^{*}$ \\
Ítem 13 & 3.19 & 0.88 & $0.48^{*}$ \\
Ítem 14 & 3.06 & 1.03 & $0.40^{*}$ \\
Ítem 15 & 2.90 & 0.81 & $0.40^{*}$ \\
Ítem 16 & 2.95 & 1.08 & $0.46^{*}$ \\
\hline
\end{tabular}

${ }^{*} \mathrm{p}<.05$

$\mathrm{n}=235$ 


\section{Escala de Procrastinación Académica}

Los resultados del análisis psicométrico de la EPA (véase la tabla 4) permiten apreciar que los ítems fluctúan entre 0.31 (ítem 7) y 0.54 (ítem 9), los cuales son estadísticamente significativos y superan el criterio de ser mayores de 0.20 (Kline, 1995), por lo que todos los ítems son aceptados. En el caso de la confiabilidad se observa que el coeficiente de consistencia interna Alfa de Cronbach asciende a 0.80 , lo que permite concluir que la EPA presenta confiabilidad.
El análisis de la validez de constructo de la EPA efectuado con la aplicación del análisis factorial exploratorio (véase la tabla 5), permite observar que:

a) La medida de adecuación del muestreo de Kaiser-Meyer-Olkin alcanza un valor de 0.80 , lo cual indica que las variables tienen un adecuado potencial explicativo.

b) El test de esfericidad de Bartlett alcanza un valor que es significativo (Chi-cuadrado $=701.95 \mathrm{p}<.05)$, lo cual indica que las correlaciones entre los ítems del instrumento son lo suficientemente elevados como para continuar con el análisis factorial.

Tabla 5

Análisis de la validez de constructo de la EPA a través del análisis factorial exploratorio

\begin{tabular}{lccc}
\hline Ítem & M & D. E. & Factor 1 \\
\hline Ítem 1 & 3.05 & 0.88 & -0.64 \\
Ítem 2 & 2.74 & 0.88 & 0.47 \\
Ítem 3 & 2.95 & 1.04 & 0.30 \\
Ítem 4 & 2.83 & 1.09 & 0.31 \\
Ítem 5 & 3.43 & 0.96 & 0.32 \\
Ítem 6 & 4.40 & 0.92 & 0.30 \\
Ítem 7 & 3.58 & 0.85 & 0.58 \\
Ítem 8 & 2.95 & 0.99 & 0.60 \\
Ítem 9 & 2.92 & 1.05 & -0.55 \\
Ítem 10 & 3.44 & 0.95 & 0.53 \\
Ítem 11 & 3.03 & 0.94 & 0.60 \\
Ítem 12 & 3.38 & 0.91 & 0.67 \\
Ítem 13 & 3.19 & 0.88 & 0.58 \\
Ítem 14 & 3.06 & 1.03 & 0.61 \\
Ítem 15 & 2.90 & 0.81 & 0.31 \\
Ítem 16 & 2.95 & 1.08 & 0.32 \\
\hline
\end{tabular}

Determinante $=0.046$

Medida de adecuación muestral de Kaiser-Meyer-Olkin. $=0.80$

Prueba de esfericidad de Bartlett Chi-cuadrado $=701.95$ G. L. $=120 p=.0001$ 
El análisis realizado a través del método de los componentes principales indica que existe un solo factor que permite explicar el $23,89 \%$ de la varianza total.

Los resultados permiten concluir que la escala presenta validez de constructo.

Adicionalmente, se realizó el análisis de la correlación entre la procrastinación general y la procrastinación académica, observándose en la tabla 6 que la correlación alcanza un valor de -0.03 que no es significativa y puede clasificarse como nula, es decir que no existe correlación significativa entre los dos aspectos evaluados.

\section{Análisis descriptivo}

Los resultados del análisis de la bondad de ajuste a la curva normal, realizados a través de la prueba de KolmogorovSmirnov (véase la tabla 7), indican que tanto en el caso de la procrastinación general como en la procrastinación académica se obtuvieron estadísticos con valores pequeños, que no presentan diferencias estadísticas significativas, por lo que se puede concluir que las distribuciones de los puntajes se aproximan adecuadamente a la distribución normal.

Tabla 6

Análisis de la correlación entre la procrastinación general y la procrastinación académica

\begin{tabular}{|c|c|}
\hline Variable & $\begin{array}{c}\text { Procrastinación académica } \\
r\end{array}$ \\
\hline Procrastinación general & -0.03 \\
\hline
\end{tabular}

$\mathrm{N}=235$

Tabla 7

Análisis de la bondad de ajuste a la curva normal de las EPG y EPA

\begin{tabular}{lccc}
\hline Variables & M & D. E. & Z de Kolmogorov-Smirnov \\
\hline Procrastinación general & 38.25 & 6.41 & 1.02 \\
Procrastinación académica & 50.82 & 4.96 & 1.15 \\
\hline
\end{tabular}

$n=235$ 
Tabla 8

Análisis de la prueba $Z$ de diferencia de medias por sexo de la EPG

\begin{tabular}{llllll}
\hline & \multicolumn{2}{c}{$\begin{array}{c}\text { Hombres } \\
\mathbf{N}=132\end{array}$} & \multicolumn{2}{c}{$\begin{array}{c}\text { Mujeres } \\
\mathbf{N}=103\end{array}$} & $\mathbf{Z}$ \\
\cline { 2 - 5 } Variable & $\mathbf{M}$ & $\mathrm{DE}$ & $\mathbf{M}$ & $\mathbf{D E}$ \\
\hline Procrastinación general & 38.77 & 6.06 & 37.50 & 6.79 & \\
\hline
\end{tabular}

Tabla 9

Análisis de la prueba $Z$ de diferencia de medias por sexo de la EPA

\begin{tabular}{|c|c|c|c|c|c|}
\hline \multirow{2}{*}{ Variable } & \multicolumn{2}{|c|}{$\begin{array}{c}\text { Hombres } \\
\mathrm{N}=132\end{array}$} & \multicolumn{2}{|c|}{$\begin{array}{l}\text { Mujeres } \\
N=103\end{array}$} & \multirow{2}{*}{$\mathbf{Z}$} \\
\hline & $\mathbf{M}$ & DE & M & DE & \\
\hline Procrastinación académica & 51.10 & 5.18 & 50.45 & 4.65 & 0.99 \\
\hline
\end{tabular}

\section{Contraste de hipótesis}

Inicialmente se planteó la hipótesis teórica, que propuso que existen diferencias en la procrastinación general por rol genérico. Para contrastarla se efectuó el análisis a través de la prueba $\mathrm{Z}$ de diferencia de medias. Al analizar la tabla 8 , se puede observar un puntaje $\mathrm{Z}=1.42$, el cual no es estadísticamente significativo $(\mathrm{p}>.05)$.

Asimismo, la media de hombres y la media de mujeres son similares, por lo que se concluye que la primera hipótesis, en la cual se planteó que existen diferencias en la procrastinación general por rol genérico, no se puede aceptar.
En lo que concierne al análisis de la hipótesis 2, la cual indica que existen diferencias en la procrastinación académica por sexo, efectuada a través de la prueba $\mathrm{Z}$ de diferencia de medias, en la tabla 9 se encuentra un estadístico $\mathrm{Z}=0.99$ que no es estadísticamente significativo $(\mathrm{p}>.05)$.

El resultado alcanzado permite concluir que la hipótesis 2 no es válida, por lo tanto las medias de los hombres y las mujeres son similares en la procrastinación académica.

Los alumnos de cuarto año y quinto año de secundaria presentan medias similares en la EPG (véase la tabla 10); 
Tabla 10

Análisis de la prueba $Z$ de diferencia de medias según grado de estudio de la EPG

\begin{tabular}{|c|c|c|c|c|c|}
\hline \multirow{2}{*}{ Variable } & \multicolumn{2}{|c|}{$\begin{array}{l}\text { Cuarto año } \\
\qquad \mathbf{N}=80\end{array}$} & \multicolumn{2}{|c|}{$\begin{array}{l}\text { Quinto año } \\
\qquad N=155\end{array}$} & \multirow{2}{*}{$\mathbf{z}$} \\
\hline & M & DE & $\mathbf{M}$ & DE & \\
\hline Procrastinación general & 38.40 & 6.40 & 38.17 & 6.42 & 0.26 \\
\hline
\end{tabular}

Tabla 11

Análisis de la prueba $z$ de diferencia de medias según grado de estudio de la EPA

\begin{tabular}{lccccc}
\hline & \multicolumn{2}{c}{$\begin{array}{c}\text { Cuarto año } \\
\mathbf{N}=80\end{array}$} & \multicolumn{2}{c}{$\begin{array}{c}\text { Quinto año } \\
\mathbf{N}=155\end{array}$} & $\mathbf{Z}$ \\
\cline { 2 - 5 } Variable & $\mathbf{M}$ & $\mathrm{DE}$ & $\mathbf{M}$ & $\mathbf{D E}$ \\
\hline Procrastinación académica & 50.13 & 4.79 & 51.17 & 5.02 & -0.55 \\
\hline
\end{tabular}

por lo tanto, la hipótesis 3 no se acepta. Asimismo, el puntaje $Z=0.26$ refiere que las diferencias no son estadísticamente significativas $(\mathrm{p}>.05)$.

Finalmente, al analizar la hipótesis 4 , los resultados indican un estadístico $\mathrm{Z}=-0.55$, que no es estadísticamente significativo $(\mathrm{p}>.05)$. Por esta razón, se concluye que esta hipótesis no es válida y que las medias de los alumnos de cuarto y quinto año de secundaria son similares en la procrastinación académica (véase la tabla 11).

\section{ANÁLISIS COMPLEMENTARIO}

Con la finalidad de establecer qué tipo de procrastinación se presentaba de forma más elevada en los alumnos, se procedió a calcular en cada escala el promedio de respuestas a los ítems. A partir de esta, se ha identificado que la incidencia de la procrastinación académica es mayor que la procrastinación general en los alumnos de cuarto y quinto año de secundaria de ambos roles genéricos, debido a que la primera $(\mathrm{M}=3.18$ D.E. $=0.31)$ presenta una mayor valoración que la última $(\mathrm{M}=2.94$ D.E. $=0.49)$ (véase la tabla 12). Así, para indagar si las diferencias son significativas se realizó la comparación de dichas medias aritméticas, obteniéndose un estadístico $\mathrm{Z}=6.08$, lo cual indica que son diferentes estadísticamente $(\mathrm{p}<.001)$. 
Tabla 12

Análisis comparativo de la procrastinación general y la procrastinación académica con valores equivalentes

\begin{tabular}{|c|c|c|c|c|}
\hline \multicolumn{2}{|c|}{ Procrastinación académica } & \multicolumn{2}{|c|}{ Procrastinación general } & \multirow{2}{*}{$\mathbf{z}$} \\
\hline $\mathbf{M}$ & DE & M & DE & \\
\hline 3.18 & 0.31 & 2.94 & 0.49 & $6.08^{* * *}$ \\
\hline
\end{tabular}

\section{DISCUSIÓN}

Los resultados obtenidos finalmente confirman lo señalado por Argumedo (2002) cuando menciona en su investigación que no se observan diferencias relevantes en procrastinación según el rol genérico, la edad y el nivel de instrucción.

El no existir diferencias entre alumnos de cuarto y quinto año de secundaria, se puede suponer que ambos grupos son muy similares en sus estrategias para enfrentar los retos académicos, incluyendo sus niveles de procrastinación. Para futuras investigaciones, se podría indagar la posible existencia de diferencias entre los alumnos que inician sus estudios secundarios y aquellos próximos a egresar, esto es, indagar si la experiencia académica influye en la variable investigada. Un similar análisis se podría realizar con la variable edad.

En lo que concierne al análisis de las estadísticas descriptivas de la procrastinación general y la procrastina- ción académica con valores equiparados, ambas escalas puestas al mismo nivel señalan que la procrastinación académica tiene mayor presencia que la procrastinación general al haberse encontrado diferencias significativas entre las medias aritméticas correspondientes. Este resultado sigue la tendencia señalada por otros investigadores, como Ellis y Knaus (1977) y O'Brien (2002; citado por Steel, 2007), quienes indican que en ambientes académicos, el porcentaje de personas que procrastinan se incrementa hasta en un 80 a $95 \%$. De esta manera, la cantidad de procrastinadores es considerable, pues los estudiantes reportan este tipo de conductas que abarcan algo más de un tercio de sus actividades diarias, a menudo a través de ocupaciones como dormir, jugar o ver televisión (Pychil, Lee, Thibodeau \& Blunt, 2000, citado por Binder, 2000).

En este trabajo no se consideró la variable social, sin embargo, sería interesante analizar la influencia de las 
características de los grupos al que pertenecen los alumnos, teniéndose en consideración los aportes de la Psicología social acerca de la influencia del grupo sobre la conducta individual.

Con lo expuesto anteriormente y siguiendo a Fee y Tangney (2000, citado por Steel, 2007), quienes afirman que si bien todas las personas procrastinan alguna vez en su vida, el $20 \%$ de la población considera la procrastinación como un problema importante que produce sentimientos de minusvalía y de falta de satisfacción en la vida en general, motivo por el cual resulta indispensable la urgente intervención de la Psicología tanto educativa como clínica, con el fin de cambiar esta situación, logrando que participen alumnos, profesores y padres de familia.

\section{CONCLUSIONES}

- La procrastinación general es igual en los alumnos de cuarto y quinto año de secundaria; no existen diferencias por grado de estudio.

- La procrastinación académica en los alumnos de cuarto y quinto año de secundaria es igual, por lo que no existen diferencias por grado académico.

- Se encontró que en ambos roles genéricos, la procrastinación general y académica es igual, por lo que no existen diferencias significativas.

- La incidencia de la procrastinación académica es mayor que la procras- tinación general en los alumnos de cuarto y quinto año de secundaria de ambos roles genéricos.

\section{REFERENCIAS}

Akinsola, M. K. (2007). Correlates of academic procrastination and mathematics achievement of university undergraduate students. Eurasia Journal of Mathematics, Science \& Technology Education, 3 (4), 363370 .

Argumedo, D. (2002). Evaluación de la confiabilidad y la estructura factorial de tres escalas de procrastinación crónica. Revista de Psicología (Pontificia Universidad Católica del Perú), 23 (1).

Balkis, M. (2009). Prevalence of academic procrastination behavior among pre-service teachers, and its relationship with demographics and individual preferences. Journal of Theory and Practice in Education, 5 (1), 18-32.

Binder, L. (2000). The effects of an academic procrastination treatment on student procrastination and subjective well-being. Tesis de maestría no publicada. Ottawa: Carleton University.

Blunt, A. (1998). Task aversiveness and procrastination: A multidimensional approach to task aversiveness across stages of personal 
projects. Tesis de maestría no publicada. Ottawa: Carleton University.

Busko, D. A. (1998). Causes and consequences of perfectionism and procrastination: A structural equation model. Tesis de maestría no publicada. Guelph, Ontario: University of Guelph.

Ellis, A. \& Knaus, W. J. (1977). Overcoming procrastination. Nueva York: Signet Books.

Ferrari, J. R. (1995). Perfectionism cognitions with nonclinical and clinical samples. Journal of Social Behavior and Personality, 10, 143-156.

Ferrari, J. \& Diaz-Morales, J. (2007). Perceptions of self concept and self presentation by procrastinators: further evidence. The Spanish Journal of Psychology, 10 (1), 91-96.

Ferrari, J. R.; Johnson, J. L. \& McCown, W. G. (1995). Procrastination and task avoidance: Theory, research, and treatment. Nueva York: Plenum Press.

Hernández, R.; Fernández, C. \& Baptista, P. (2006). Metodología de la investigación. México, D.F.: McGraw-Hill/Interamericana.

Kline, P. (1995). The handbook of psychological testing. Londres: Routledge.
LaForge, M. (2005). Applying explanatory style to academic procrastination. Journal of the Academy of Business Education, 6, 1-7. < http:// www.abe.sju.edu/proc2005/laforge.pdf $>$. [Consulta: Agosto del 2009.]

Rothblum, E. D.; Solomon, L. J. \& Murakami, J. (1986). Affective, cognitive, and behavioral differences between high and low procrastinators. Journal of Counseling Psychology, 33, 387-394.

Siegel, S. \& Castellan, N. J. (1995). Estadística no paramétrica aplicada a la ciencia de la conducta. México, D.F.: Trillas.

Skinner, B.F. (1977). Ciencia y conducta humana. Barcelona: Fontanella.

Solomon, L. J. \& Rothblum, E. D. (1984). Academic procrastination: Frequency and cognitive-behavioral correlates. Journal of Counseling Psychology, 31, 503-509.

Steel, P. (2007). The nature of procrastination: A meta-analytic and theoretical review of quintessential selfregulatory failure. Psychological Bulletin, 133 (1), 65-94.

Wolters, C. A. (2003). Understanding procrastination from a self-regulated learning perspective. Journal of Educational Psychology, 95, 179-187. 


\section{ANEXO 1 \\ ESCALA DE PROCRASTINACIÓN GENERAL}

Deborah Ann Busko (1998)

Adaptación: Óscar Álvarez

Sexo:

Edad:

Grado:

\section{Intrucciones}

A continuación se presenta una serie de enunciados sobre su forma de estudiar, lea atentamente cada uno de ellos y responda (en la hoja de respuestas) con total sinceridad en la columna a la que pertenece su respuesta, tomando en cuenta el siguiente cuadro:

\begin{tabular}{|cl|}
\hline$S$ & SIEMPRE (Me ocurre siempre) \\
CS & CASI SIEMPRE (Me ocurre mucho) \\
A & A VECES (Me ocurre alguna vez) \\
CN & POCAS VECES (Me ocurre pocas veces o casi nunca) \\
$N$ & NUNCA (No me ocurre nunca) \\
\hline
\end{tabular}

\begin{tabular}{|c|c|c|c|c|c|c|}
\hline $\mathbf{N}^{\circ}$ & Ítem & $\mathbf{S}$ & CS & A & $\mathrm{CN}$ & $\mathbf{N}$ \\
\hline 1 & $\begin{array}{l}\text { Cuando tengo una fecha límite para hacer algo, espero hasta } \\
\text { el último minuto para hacerlo }\end{array}$ & & & & & \\
\hline 2 & Encuentro una excusa para no hacer lo que tenía que hacer & & & & & \\
\hline 3 & Tiendo a perder mucho el tiempo & & & & & \\
\hline 4 & Aplazo la toma de decisiones difíciles & & & & & \\
\hline 5 & Casi siempre llego a tiempo a mis reuniones & & & & & \\
\hline 6 & Cuando me canso de hacer una tarea, tiendo a aplazarla & & & & & \\
\hline 7 & Cuando me aburro de una tarea, tiendo a aplazarla & & & & & \\
\hline 8 & Me disgusta seguir pautas estrictas & & & & & \\
\hline 9 & $\begin{array}{l}\text { Sin tener que desarrollar otra actividad importante, aplazo } \\
\text { realizar una tarea }\end{array}$ & & & & & \\
\hline 10 & No puedo cambiar mi hábito de perder el tiempo & & & & & \\
\hline 11 & Tiendo a descuidar las tareas que me son muy difíciles & & & & & \\
\hline 12 & $\begin{array}{l}\text { Me distraigo fácilmente cuando trato de concentrarme en } \\
\text { hacer algo }\end{array}$ & & & & & \\
\hline 13 & Cuando tomo una decisión, la cumplo & & & & & \\
\hline
\end{tabular}




\section{ANEXO 2}

ESCALA DE PROCRASTINACIÓN ACADÉMICA

Deborah Ann Busko (1998)

Adaptación: Óscar Álvarez

Sexo:

Edad:

Grado:

\section{Intrucciones}

A continuación se presenta una serie de enunciados sobre su forma de estudiar, lea atentamente cada uno de ellos y responda (en la hoja de respuestas) con total sinceridad en la columna a la que pertenece su respuesta, tomando en cuenta el siguiente cuadro

\begin{tabular}{|cl|}
\hline S & SIEMPRE (Me ocurre siempre) \\
CS & CASI SIEMPRE (Me ocurre mucho) \\
A & A VECES (Me ocurre alguna vez) \\
CN & POCAS VECES (Me ocurre pocas veces o casi nunca) \\
N & NUNCA (No me ocurre nunca) \\
\hline
\end{tabular}

\begin{tabular}{|c|c|c|c|c|c|c|}
\hline $\mathbf{N}^{\circ}$ & Ítem & S & CS & A & CN & $\mathbf{N}$ \\
\hline 1 & $\begin{array}{l}\text { Cuando tengo que hacer una tarea, normalmente la dejo para el } \\
\text { último minuto }\end{array}$ & & & & & \\
\hline 2 & Generalmente me preparo por adelantado para los exámenes & & & & & \\
\hline 3 & Cuando me asignan lecturas, las leo la noche anterior & & & & & \\
\hline 4 & Cuando me asignan lecturas, las reviso el mismo día de la clase & & & & & \\
\hline 5 & $\begin{array}{l}\text { Cuando tengo problemas para entender algo, inmediatamente } \\
\text { trato de buscar ayuda }\end{array}$ & & & & & \\
\hline 6 & Asisto regularmente a clases & & & & & \\
\hline 7 & Trato de completar el trabajo asignado lo más pronto posible & & & & & \\
\hline 8 & Postergo los trabajos de los cursos que no me gustan & & & & & \\
\hline 9 & Postergo las lecturas de los cursos que no me gustan & & & & & \\
\hline 10 & Constantemente intento mejorar mis hábitos de estudio & & & & & \\
\hline 11 & $\begin{array}{l}\text { Invierto el tiempo necesario en estudiar aun cuando el tema sea } \\
\text { aburrido }\end{array}$ & & & & & \\
\hline 12 & Trato de motivarme para mantener mi ritmo de estudio & & & & & \\
\hline 13 & Trato de terminar mis trabajos importantes con tiempo de sobra & & & & & \\
\hline 14 & Me tomo el tiempo de revisar mis tareas antes de entregarlas & & & & & \\
\hline 15 & Raramente dejo para mañana lo que puedo hacer hoy & & & & & \\
\hline 16 & $\begin{array}{l}\text { Disfruto la mezcla de desafío con emoción de esperar hasta el } \\
\text { último minuto para completar una tarea }\end{array}$ & & & & & \\
\hline
\end{tabular}

